Nouvelles perspectives en sciences sociales

\title{
Territoires de l'action publique spatiale et espaces réticulaires individuels : imbrication problématique
}

\section{Denis Martouzet}

Volume 4, numéro 1, septembre 2008

URI : https://id.erudit.org/iderudit/019641ar

DOI : https://doi.org/10.7202/019641ar

Aller au sommaire du numéro

\section{Éditeur(s)}

Prise de parole

\section{ISSN}

1712-8307 (imprimé)

1918-7475 (numérique)

Découvrir la revue

\section{Citer cet article}

Martouzet, D. (2008). Territoires de l'action publique spatiale et espaces réticulaires individuels : imbrication problématique. Nouvelles perspectives en sciences sociales, 4(1), 107-139. https://doi.org/10.7202/019641ar

\section{Résumé de l'article}

L'auteur examine les difficultés mais aussi les possibilités de prise en compte des espaces vécus pour l'élaboration de projets de territoire dans le cadre de la participation du public au projet. Les spatialités individuelles, connues selon une méthode d'enquête originale et réévaluant la notion d'espace vécu par celle d'espaces dits, n'ont ni une échelle ni une structure pouvant correspondre aux territoires institutionnels de l'action publique. L'habiter des individus est défini comme le mode et le contenu des justifications d'évocations d'espaces et de lieux fréquentés ou simplement pensés, justifications dont les différents types (qualification, explication, rationalisation) sont précisés. Cet habiter permet de distinguer, de façon archétypale, les individus qui se réfèrent prioritairement à un ou des modèles spatiaux construits pendant leur vie, de ceux qui, par opposition, se réfèrent à un ou des modèles de type relationnel (deux exemples sont détaillés). Ceux des premiers peuvent être pris en compte par les urbanistes, les autres beaucoup plus difficilement. 


\title{
Territoires de l'action publique spatiale et espaces réticulaires individuels : imbrication problématique
}

\author{
Denis Martouzet \\ Université François-Rabelais, Tours
}

\section{Introduction}

T objectif premier de ce texte consiste en la mise en évidence $\mathcal{L}$ du décalage qui existe entre espace vécu et territoire de projet. Nous proposons de montrer la réalité de l' "érosion de la logique territoriale " par le « développement des réseaux », réseaux construits et développés par les individus eux-mêmes. La dimension réticulaire de la spatialité d'un individu, que ce soit à l'échelle de la vie de l'individu ou sur des durées moins étendues, met à mal les politiques territoriales dans la mesure où elle est changeante et ne répond que très partiellement à des entités spatiales qui pourraient préfigurer autant de territoires. Le présent propos est l'un des résultats d'une recherche intitulée Espaces habités, espaces anticipés (EHEA), financée par l'Agence Nationale de la Recherche, menée entre janvier 2006 et mars 2008, et plus particulièrement le module 1 Espaces habités, qui a visé à vérifier que les multiples pratiques spatiales des individus, de la fréquen-

1 Pour reprendre les termes de l'appel à communication du CR05 pour le congrès quadri-annuel de l'Association internationale des sociologues de langue française (AISLF) de 2008. 
tation de tel ou tel lieu, aux choix de localisation résidentielle, en passant par les objets permettant les déplacements, etc., constituent un rapport construit à l'espace qui permet à l'individu d'en qualifier, explicitement ou non, les composants et les différents types ${ }^{2}$ (collectif EHEA, 2008). Cette qualification dépend de multiples facteurs, de la trajectoire d'une vie à son environnement social : l'hypothèse avancée est que cette qualification, qui fait que certains espaces et types sont plus affectionnés que d'autres, que certains peuvent faire l'objet d'une déqualification, voire même d'un rejet, tant pour des raisons personnelles que liées à des événements sociétaux, est un élément qui a autant d'importance si ce n'est plus, que d'autres, dans les choix contribuant à la constitution des espaces habités d'un individu. Cela induit une conception spécifique de l'espace, qui en fait un $\operatorname{actant}^{3}$ ou un ensemble d'actants, que l'on peut définir par la complémentarité qui lie acteur (celui qui agit) et actant comme tout élément qui fait (ré)agir. Ce rapport construit fait que l'espace n'est pas un simple support, mais qu'il est pleinement chargé de sens, engagé dans la réalisation des pratiques spatiales. Ce rapport, que l'on pourrait aussi qualifier d'affectif, en complément à sa dimension fonctionnelle, n'est pas que l'affaire de l'habitant ordinaire, sans doute aussi l'est-il de la part des décideurs et professionnels de l'aménagement, comme les architectes, les urbanistes, les aménageurs, etc., mais nous aborderons ici cette qualification de l'espace par l'habitant.

Des résultats obtenus, nous extrayons certains aspects montrant, d'une part, ce décalage, qui est l'incommensurabilité entre l'espace individuel et le territoire collectif ou politique et, d'autre part, la possibilité pour les acteurs de l'aménagement et de l'organisation du territoire de réduire ce décalage, dans certains cas et sous certaines conditions, par la prise en compte de certaines caractéristiques relatives au parcours de l'individu.

L'exposé préalable de deux constats est nécessaire. Tout d'abord

$2 \quad$ Collectif EHEA, Espaces habités, espaces anticipés, rapport de recherche UMR CITERES pour le compte de l'agence nationale de la recherche, 2008.

3 Jacques Lévy et Michel Lussault (dir.), Dictionnaire de la géographie et de l'espace des sociétés, Paris, Belin. 
l'organisation du territoire national et ses déclinaisons locales à quelque échelle que ce soit vise un objectif, assez peu clairement défini, de cohérence. Cet objectif que l'on peut qualifier d'inhérent à l'aménagement devient une valeur opérationnelle de l'action en aménagement, en en faisant un objectif peu remis en cause, sans cesse explicitement ou implicitement évoqué, voire invoqué, valeur concrétisée par les lois d'organisation du territoire (LOADT, LOADDT, SRU) ${ }^{4}$ avec notamment l'invention du Schéma de Cohérence Territoriale. On peut, sans trop forcer le trait, faire de l'histoire de l'intercommunalité celle de la recherche de la cohérence territoriale, avec son corollaire, la recherche sans cesse renouvelée et toujours problématique du périmètre d'action pertinent. La définition même de la cohérence pose problème, fait l'objet de débats tant théoriques que pratiques, à l'occasion de l'élaboration de chaque document de planification. $\mathrm{Ni}$ trop homogène, sous peine d'uniformité peu propice à un projet, ni trop hétérogène, un territoire cohérent doit, semble-t-il, être composé d'éléments spatiaux complémentaires, c'est-à-dire à la fois différents et se complétant réciproquement, pour que l'ensemble - le territoire - réponde aux aspirations et aux besoins des acteurs locaux (habitants, entreprises, autres institutions notamment), en interaction avec l'emboîtement de territoires plus vastes l'englobant, ce qu'on appelle projet de territoire faisant de ce territoire un territoire de projet(s), l'un et l'autre théoriquement se co-construisant.

Le second constat nous place du côté des habitants invités et incités à participer à l'élaboration des documents de planification et, ce faisant, du projet de territoire. Bien que pouvant avoir une conception " experte " de l'organisation du territoire ou une approche idéologique de celle-ci, l'habitant ne peut pas ne pas être influencé par son expérience personnelle du territoire et de son fonctionnement. Son expérience, ses pratiques sont bien

$4 \quad$ Loi d'Orientation de l'Aménagement et du Développement du territoire, Loi $\mathrm{N}^{\circ}$ 95-115 du 4 février 1995, dite Loi Pasqua; Loi d'Orientation pour l'Aménagement et du Développement Durable du Territoire, Loi No 99-533 du 25 juin 1999, dite Loi Voynet; Loi Solidarité et Renouvellement Urbains, Loi $\mathrm{N}^{\circ} 2000-1208$ du 13 décembre 2000. 
souvent ses principales sources d'information concernant les aspects les plus concrets de son territoire, les aspects qui le touchent le plus. C'est ce que les géographes nomment espace vécu. Or, nous montrons que cet espace pratiqué est aux antipodes du territoire de projet dans lequel l'habitant est, de fait, inclus.

Cet habitant est inscrit dans la tendance générale d'une individualisation de la personne $e^{5}$ qui se concrétise par le fait que chacun, plus que pour les générations passées, peut se construire par diverses formes de multi-appartenance, évolutives et distinctes de celle d'autrui, multi-appartenance où la contrainte spatiale est moins prégnante. Les trajectoires de vie se font de plus en plus à la carte, que ce soit sur le plan familial, professionnel, affectif ou autre, que ce soit dans les activités individuelles ou collectives. Les choix deviennent très ouverts, les trajectoires se différencient d'un individu à l'autre et évoluent pour une même personne. Ceci a des répercussions spatiales ou, inversement, les comportements spatiaux montrent cette évolution sociale : augmentation du nombre, de la fréquence et de la longueur des déplacements, augmentation de la variabilité de ces variables par unité de temps, augmentation de la diversité des motifs des déplacements, plus grande variabilité des moments de déplacement, que ce soit à l'échelle de la journée, à celle de la semaine, des saisons ou de l'année. Ceci est aussi rendu possible par l'accroissement de l'instabilité professionnelle, par les variations relatives des coûts des déplacements, par les recompositions familiales et donc l'élargissement géographique de la famille, par le fait que la ville soit disponible 24 heures sur $24^{6}$.

La recherche de cohérence et les aspects aléatoires et imprévisibles des pratiques individuelles de l'espace sont à l'origine de ce décalage. Nous montrerons d'abord, après quelques points d'ordre méthodologique précisant la notion d'espace vécu par celle d'espace dit, l'ampleur de l'extension spatiale de ce phénomène pour ensuite aborder les justifications qui sont apportées à

\footnotetext{
Voir, par exemple, Jean-Claude Kaufman, Linvention de soi, une théorie de l'identité, Paris, Armand Colin, 2004.

$6 \quad$ Luc Gwiazdzinski (dir.), La ville 24 heures sur 24 : regards croisés sur la société en continu, La Tour d'Aigues, Éditions de l'Aube, Paris, DATAR, 2003.
} 
la fréquentation de ces lieux, ce que nous nommons spatialité, et les qualifications qui sont données à ces lieux, qui forment l'habiter d'un individu. Enfin, deux exemples donneront des indications sur les possibilités de prise en compte, dans la participation, des espaces dits pour un projet territorial.

\section{Habiter, espaces dits et leurs représentations}

\subsection{Habiter et spatialité}

Étant donné le protocole d'enquête dont nous ne présenterons ici que les grandes lignes, nous sommes dans la nécessité de distinguer ce que nous nommons les espaces dits des espaces vécus, notion plus couramment utilisées en recherche en sciences sociales et, de façon extensive, dans la pratique de l'aménagement et de l'urbanisme comme pseudo-soubassement théorique à la légitimation, au moins partielle, de la participation des habitants qui le souhaitent à l'élaboration des projets spatiaux et, notamment, au projet de « leur» territoire.

L'objectif du module 1 de cette recherche visait donc à connaître l'habiter d'un individu à travers sa construction au cours de sa vie, c'est-à-dire ce qui, dans son passé notamment mais aussi dans sa situation actuelle et son avenir tel qu'il est représenté au présent, permet de comprendre, au moins en partie, la nature, la plus précise possible, de son rapport à l'espace. Autrement dit, par habiter ${ }^{7}$, nous entendons tout ce qui fait qu'un individu est là et pas ailleurs ou voudrait ne pas y être et être ailleurs ou veux être là et pas ailleurs, etc. C'est l'argumentation (l'ensemble des justifications apportées : qualifications, explications, rationalisations et évaluations) concernant les lieux qui permet de passer de la spatialité, objective et factuelle, à l'habiter, unique, subjectif, évolutif et de l’ordre de l'idéel.

$\overline{7}$ On devrait, en accord avec Thierry Paquot notamment, distinguer l'habiter (substantif) d'habiter (verbe), dans la mesure où le premier définit le rapport à l'espace, tandis que le second définit le fait d'être en accord avec ce rapport à l'espace. Ici, il s'agira sauf indication contraire du substantif. Voir Thierry Paquot, Michel Lussault et Chris Younès (dir.), Habiter, le propre de l'homme. Villes, territoires et philosophie, Paris, La Découverte, 2007. 
La définition du concept d'habiter, celle de son contenu font cependant l'objet de débats, par exemple lors du colloque $H_{a b i t e r}{ }^{8}$ qui a préfiguré l'ouvrage Habiter, le propre de l'homme'. Il apparaît que l'habiter dont nous traitons ici, et qui serait celui des sciences de l'espace ou plus largement des sciences sociales, est à distinguer de l'habiter philosophique et notamment heideggerien qui est un rapport au monde sans commune mesure spatiale ou temporelle avec l'habiter pratique que nous mobilisons ici. Le concept d'habiter tel qu'utilisé par Martin Heidegger ${ }^{10}$ réfere à un rapport au monde en tant qu'entité cohérente, unique et dynamique, une échelle globale et non locale. Il ne réfère donc pas à un espace en particulier de ce monde, ni à un système d'espaces et de lieux particuliers, même si ce système, cet habiter géographique construisent l'image que l'on a du monde. D'autre part, habiter dans le sens d' "être-déjà-là » ou d' " être-toujoursdéjà-là " renvoie à une temporalité particulière ou à une absence de temporalité, ce qui ne permet pas de supposer une construction du même ordre de l'habiter. Chez Heidegger, habiter est une façon d'être ou, dans sa dimension éthique, une façon de devoirêtre face et dans le monde. Celui-ci préexiste, tandis que nous partons de l'idée que, concernant notre habiter pratique, notre monde se construit, il n'est donc pas " en-soi ». Ces deux approches ne sont pas contradictoires au point de s'exclure, mais apparaissent comme complémentaires, l'une plus philosophique dont la dimension essentielle est éthique, l'autre plus sociopsychologique dont la dimension essentielle est descriptive. Il ne s'agira donc pas d'explorer systématiquement ce que signifie " habiter " comme le fait Augustin Berque ${ }^{11}$ sur le plan étymo-

8 Colloque « Habiter » organisé conjointement par le laboratoire GERPHAU, 1 UMR CNRS 7145 LOUEST de l'École nationale supérieure d'architecture de Clermont-Ferrand, l'École nationale supérieure d'architecture de Bretagne (Rennes), l'Institut d'urbanisme de Paris, l'Université François-Rabelais de Tours (MSHS Villes et territoires et UMR 6173 CITERES), 11-12 mai 2006, Créteil.

9 Thierry Paquot, Michel Lussault et Chris Younès (dir.), Habiter, le propre de l'homme..., op. cit.

10 Martin Heidegger, Essais et conférences, Paris, Gallimard, 1958.

11 Augustin Berque, "Qu'est-ce que l'espace de l'habiter? "Colloque, IUP, 
logique, ou comme on pourrait le faire sur le plan philosophique, mais simplement de dire ce qu' " habiter " signifie lorsqu' on le définit comme le rapport qu'entretient l'individu avec l'espace.

\section{2. "Mais qu'est-ce que vous faites là? »}

Cet habiter pratique de l'individu a pu être questionné en demandant : "Mais qu' est-ce que vous faites là? " " ${ }^{12}$, l'enjeu ayant été d'obtenir une réponse de la part d'une série de personnes. La formulation de cette question répond de manière concrète à un ensemble d'objectifs :

Le « mais " introduit une feinte surprise de la part de l'enquêteur, comme si les réponses apportées à la question n'allaient pas de soi, n'étaient pas complètes, comme si les réponses exigeaient une autre question de type " mais, pourquoi? " (possiblement entendue comme " pour quoi? "), entraînant une autre réponse qui, elle-même, est ré-interrogée, etc., jusqu’à ce que ces itérations ne puissent plus rien amener de nouveau et finalement que l'ensemble des réponses ainsi données s'avère être explicité, après validation par la personne elle-même ou fermeture et auto-validation du système des réponses. Il s'agit, en fait, de pousser la personne dans ses retranchements, pour connaître la cause de la cause, voire la cause de la cause de la cause, etc. ou la raison de la raison, etc. dans le but de dépasser, à la fois, les discours convenus et les raisons rationnelles ${ }^{13}$. L'objectif du " mais " est ainsi double, entre établir une rupture quant à l'évidence des

11-12 mai 2006.

12 Il va de soi que ce n'est pas cette question ainsi formulée qui a été posée pendant les interviews, ce qui n'aurait pas manqué de désarçonner les personnes, mais cette formulation synthétise à la fois l'objectif de l'enquête et l'ensemble des questions posées aux différentes personnes.

13 Nous utilisons l'expression " raison rationnelle ", qui peut paraître pléonastique, par différenciation avec l'expression " raison affective " qui, elle, peut paraître paradoxale. Cette distinction est archétypale dans la mesure où, par là, nous souhaitons indiquer qu'il n'y a pas d'opposition radicale entre rationalité et affectivité, même s'il est pratique de les distinguer à condition que cette distinction soit perçue comme une construction intellectuelle visant à une certaine clarté du discours. Dans toute action, il y a une part de rationalité et une part d'affectivité et il ne s'agit pas de fonder une limite entre les deux. 
réponses induites, dans un premier temps, par le questionnement et, engager l'individu sur la voie d'un entretien exploratoire approfondi, dans le but de sonder son point de vue, ses sentiments, la signification profonde qu'il attribue à cette dimension spatiale.

Le « là » est large et pluriel, laissé au libre choix de la personne, lors de l'entretien : dans ce logement, dans ce quartier, dans cette ville, etc. La référence spatiale contenue dans l'adverbe de lieu est volontairement soumise à l'arbitrage de l'enquêté : il choisit le ou les chapitres qu'il préfère d'Espèces d'espaces de Georges Pérec. Ce "là " ouvre alors la discussion sur l'ensemble des considérations spatiales relatives aux échelles, aux connexions, aux lieux et aux liens qui forment les territoires et les réseaux de l'individu.

Le « vous » a pour fonction essentielle d'assigner le " faites là » à l'individu. C'est bien de lui et de son vécu spatial dont il s'agit. Le pronom personnel pose d'emblée le locuteur comme référent. Ce qui est ainsi visé, c'est bien le regard subjectif de l'individu sur son espace, sa vision, son interprétation des faits spatiaux et sociaux qui ont jalonné son existence. Dans ce sens, l'individu devient un objet à part entière de l'investigation, donnant forme à une nouvelle question sous-entendue mais néanmoins de prime intérêt : " mais qui êtes-vous (là)? ». Son « je » devient alors, tout autant que le " là », le sujet du discours sur l'espace.

Le "faites " est formulé de façon à ce que l'on puisse y entendre effectivement le verbe "faire ", mais aussi " être ", " rester ", " habiter ", largement au-delà du fait que la personne, en situation d'entretien, est justement en train de répondre à l'enquêteur. Il est l'expression de cet habiter concret (même s'il réfère aussi à des souvenirs, des craintes, des attentes...) distinct de l'habiter philosophique. "Faire " est un " être-là » contextualisé. Le faire est la résultante de la coordination du "vous » et du " là » mais il en est aussi l'origine : il construit le " vous " grâce au " là » et justifie ce " là " par ce qu'il permet à "je » de " faire ».

Enfin, la formule interrogative " qu'est-ce que...» implique 
" pour quelle(s) raison $(s)^{14}$ êtes-vous là? Quel est l'intérêt pour vous d'être là aujourd'hui, dans vos activités et dans ce que propose votre espace? " Mais aussi « qu'est-ce qui vous a poussé(e) ou amené(e) dans le passé à être là aujourd'hui? " et " qu'est-ce que vous prévoyez, projetez, envisagez, fantasmez pour l'avenir qui permet de comprendre pourquoi vous êtes là? ». Le " qu'estce que..." renvoie aux potentialités matérielles et symboliques de l'espace en question et leur adéquation avec les attentes et les besoins de l'individu. Il est aussi le lien entre l'enquêteur et l'enquêté, l'initiation et le principe de l'entretien.

\subsection{Situations individuelles et tranches de vie}

Les réponses obtenues s'insèrent dans deux objets-types qui permettent, en une première approche, de classer les éléments de réponse à " qu'est-ce que vous faites là? » et qui le justifient ${ }^{15}$. Le premier de ces deux objets types sont les " tranches de vie ", dont la somme, sur le plan de la durée, est égale à l'ensemble de la vie d'un individu; elles correspondent au découpage que l'on peut en faire a posteriori du récit et dont le passage d'une tranche à l'autre est marqué par un changement important de fréquentation des lieux et des déplacements, ce qui se concrétise par un déménagement ou un changement de lieu de travail. Elles correspondent à des ensembles de moments vécus par l'individu dans son passé proche et lointain et dont certains peuvent permettre de comprendre une partie de la réponse à " mais, qu'estce que vous faites là? ». On a pu inclure aussi une tranche de vie future, dont les bornes temporelles ne peuvent être fixées a priori et qui contient les événements attendus, qu’ils soient craints ou espérés. Ce lien entre passé, présent et futur, passe par des investigations en rapport avec les émotions (le rapport affectif à l'espace), les phénomènes identitaires (l'identification à l'espace), mais aussi par l'apprentissage et, enfin, par des trajectoires dont certains éléments découleraient " logiquement "d'éléments de

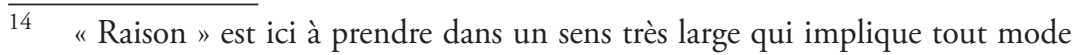
de justification, englobant tout à la fois les raisons rationnelles, les raisons affectives et les causes extérieures.

15 Ce terme « justifier » est décliné plus loin. 
vie antérieurs. Ce sont, notamment, ces trajectoires que nous voulons mettre en avant ici.

Le second objet est la "situation ». C'est le contexte actuel de la tranche de vie actuelle : quels sont les éléments extérieurs à l'individu qui expliquent sa situation, notamment par la contrainte qu'ils imposent à l'individu (mais aussi pour des raisons autres que des contraintes mais découlant d'autres choix)? La situation est l'ici et le maintenant qui environne l'individu, alors que les tranches de vie sont, de façon dynamique, l'individu, considéré de façon diachronique, dans ce qu'il a de passé et d'à venir, qui construisent l'individu d'aujourd'hui.

Par exemple, $\mathrm{M}$. X réside à proximité du littoral, d'une part, parce qu'il a habité dans son enfance près du littoral et qu'il cherche à retrouver des impressions passées, qu'il s'identifie à cet espace. Mais s'il habite près de ce littoral, c'est parce que, d'autre part, sa situation actuelle lui permet d'être en ce lieu qu'il a choisi ou ne lui permet pas d'être en un autre lieu littoral ${ }^{16}$. Cet exemple montre d'ailleurs que la distinction effectuée entre " situation " et " tranche de vie " est assez formelle puisque les deux sont en interaction constante et la délimitation entre la tranche de vie et la situation qui l'environne est relativement floue, y compris pour l'individu lui-même.

Les rapports entre les tranches de vie passées et la situation actuelle peuvent être schématisés, pour un individu, suivant les principes d'articulation présentés dans la figure 1. Cette perspective schématique permet d'inscrire la situation actuelle de l'individu dans une perspective temporelle élargie à l'échelle de sa vie, et de faire ressortir ainsi les dynamiques constitutives de son habiter.

$\overline{16}$ Systématiquement, plusieurs échelles s'imbriquent dans le fait d'être là : il s'agit en même temps d'être en ce lieu précis et dans ce type de lieu, le particulier, contingent, répondant plus ou moins exactement à la recherche d'un type. 


\section{Figure 1}

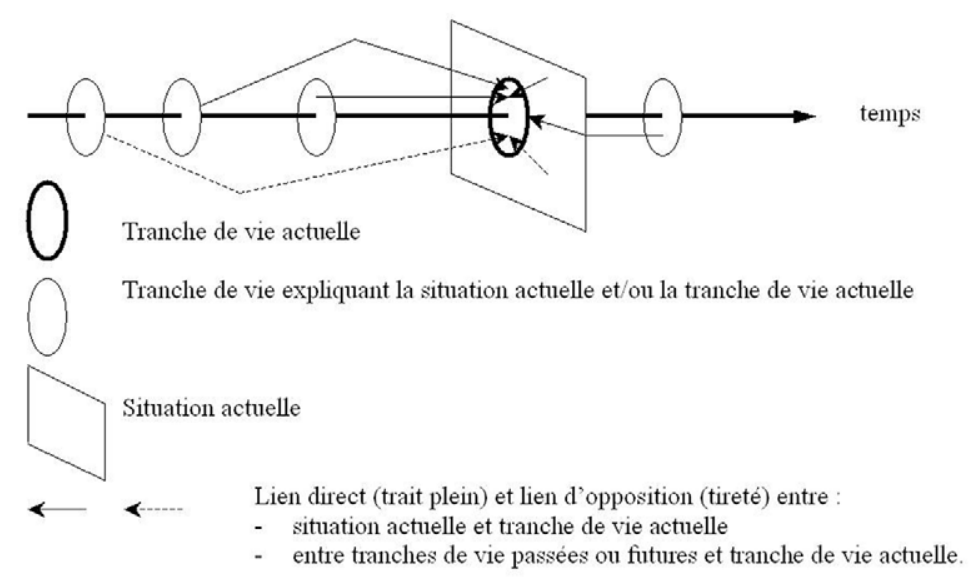

\subsection{Espaces dits et espaces vécus}

Notre échantillon, restreint du fait du caractère expérimental de la recherche menée et de la lourdeur du protocole, était composé de 12 personnes ayant entre 24 et 66 ans, de classes moyennes ou moyennes supérieures, habitant trois quartiers de l'agglomération tourangelle (quartier Colbert en centre ville de Tours, quartier des Deux-Lions en périphérie de ce centre ville et quartier Rolland-Pilain dans une commune de première couronne de l'agglomération). Il est bien évident que les caractéristiques de ces personnes rejaillissent sur les résultats obtenus. Cependant, les deux exemples que nous développons plus loin permettent d'envisager deux cas archétypaux en ce qui concerne la possibilité de prise en compte des espaces vécus, à travers les espaces dits, dans la participation au projet.

À ces 12 personnes, il a donc été demandé de faire leur récit de vie en précisant autant que possible les lieux qui ont alors été fréquentés, de la façon la plus exhaustive possible. Pour accentuer le caractère spatial de ce récit de vie, les relances se sont opérées non par le "pourquoi? " (par exemple, "pourquoi avez-vous divorcé? ") mais par le " où?? ou le " et alors? " (" et alors, suite à ce divorce, qu'avez-vous fait, où êtes-vous allé? »). 
Un quadruple tri effectué dans l'information que dispense la personne dans le contexte de l'entretien sous-tend l'écart entre espaces vécus et espaces dits. Il y a, tout d'abord et très classiquement, le tri opéré par la mémoire qui, au cours de la vie de l'individu, l'a amené à évacuer certains événements, à donner de l'importance à d'autres, à se représenter et donc modifier, voire créer certains souvenirs ${ }^{17}$, tri de la mémoire auquel il faut adjoindre celui de la mobilisation. Cette dimension mémorielle s'inscrit dans un système dynamique complexe, entre capacité d'engrammation, sélectivité, remémoration et représentation. En effet, l'individu n'est ni hypermnésique, ni totalement conditionné par son vécu, mais les éléments de données informatives qu'il est en mesure d'engranger et de restituer dépendent, à l'image de la métaphore informatique, à la fois des données qu'il aura lui-même sélectionnées pour être stockées, ainsi que du programme auquel il fait appel pour se les remémorer ${ }^{18}$. Faisant intervenir à la fois des critères de sélectivité et de représentation, le couple mémoire/mobilisation apparaît comme le filtre qui permet d'obtenir ce qui est à prendre au sérieux : le passage de ce filtre est " preuve " d'importance, de pertinence aux yeux mêmes de l'interviewé (et non preuve de vérité).

La durée limitée de l'entretien est un deuxième tri, qui renforce le premier, en contraignant la mobilisation par l'exigence que donne l'enquêteur de respecter un certain délai (une durée sur un plan quantitatif) et l'exigence qu'il donne sur un plan qualitatif en indiquant l'objet de la recherche et la nature de l'information recherchée. La personne qui a accepté d'être interrogée (et qui peut se désister à tout moment y compris une fois que l'entretien est terminé) ne peut pas ne pas répondre au moins en partie à cette exigence qualitative. Ce deuxième tri, d'origine technique, prépare le troisième qui, lui, porte sur le contenu. Ce troisième phénomène de tri, conscient ou non, que met en œuvre

17 Elizabeth Loftus, "Our changeable memories: Legal and practical implications ", Nature Review Neuroscience, no 4, 2003, p. 231-234. Jean-Claude Tabary (dir.), Praxis et cognition, Lyon, L'Interdisciplinaire, 1992, p. 95-102. 
la personne vise à lui permettre de sauvegarder tout ou partie de son intimité, par des omissions plus ou moins volontaires ou par des informations supplémentaires dont le but est de cacher à l'interlocuteur ce qui a, aux yeux de la personne enquêtée, vraiment de l'importance.

Enfin, un quatrième tri structure l'ensemble du récit, c'est celui qui veut que, en parallèle de sauvegarder son intimité, la personne cherche à garder, à montrer et à se montrer à elle-même sa cohérence, notamment sa cohérence sur le plan psychologique (il ne faut pas apparaître comme quelqu'un mené par les circonstances, ni comme quelqu' un de versatile). Cela se traduit par une cohérence formelle du discours telle que ce qui a été dit contraint ce qui va être dit : l'ensemble des " possibles à dire » des instants $\mathrm{T}_{\mathrm{n}+\mathrm{x}}$ est prédéterminé par ce qui a été dit à $\mathrm{T}_{\mathrm{n}}$ et avant. De même l'ensemble des " possibles à dire " à $T_{n}$ est, en partie, déterminé par ce qui va être dit par la suite, pour peu qu'il y ait une quelconque forme d'anticipation du contenu du discours à venir. L'ensemble, qui se traduit par cette recherche de cohérence, est sous-tendu par l'idée (plus ou moins précise et plus ou moins consciente) répondant à : « quelle image vais-je (dois-je) donner de moi-même? ». Ce quatrième tri s'impose au premier, dans sa dimension mobilisation qui est donc déterminé non par ce qui est retenu ou non retenu mais par ce qui est «à retenir pour... » ou «à ne pas retenir pour... " ${ }^{19}$.

Ainsi, les espaces dits sur lesquels nous avons travaillé sont les espaces vécus qui ont été préalablement mémorisés, mobilisés lors de l'entretien et dicibles autant que la phase de réactivation le permette. Cette phase permet de transférer certains des élé-

19 Pour contourner ces effets liées au rapport qu'entretient l'individu avec luimême et avec l'enquêteur, nous avons mis au point une méthode originale de réactivation (Hélène Bailleul, Benoît Feildel, Lise Gaignard et Denis Martouzet, «La carte : fonctionnalité transitionnelle et dépassement du récit de vie ", Nature, Sciences, Sociétés, 2009, à paraître) : la présentation du spatiogramme (que nous présenterons plus loin) à la personne elle-même, l'amène à chercher à améliorer cette représentation cartographique non pas tant par des justifications rationalisantes (par le mode de la causalité, de la finalité, par exemple) mais en laissant échapper, par le mode des associations d'idées, des raisons qui sont de l'ordre de l'affect ou de l'émotion. 
ments de justification ou plus largement certains des souvenirs du non-dit, voire de l'indicible, vers le dicible et, par suite, le dit par le changement de contexte et d'interlocuteur ${ }^{20}$. De ce fait, nous sommes dans une approche pragmatique pour laquelle ce qui est dit est à prendre au sérieux par l'enquêteur, car important pour l'enquêté et, en même temps, ce qui est important est dit.

1.5. Extension spatiale des espaces dits

Nous présentons ici l'extension géographique des spatialités de l'ensemble des 12 personnes interrogées, aux échelles internationales, nationales, infradépartementale et locale (approximativement l'échelle de l'agglomération de Tours). Ce qui ressort premièrement est l'extrême centralité des déplacements marqués par la forme en étoile de ce graphe (cartes 1 et 2), puis, à mesure que l'on s'approche des échelles les plus fines, celles du local, une image beaucoup plus maillée de ce réseau. Pour la plupart, le logement ou le couple composé du logement et du lieu de travail reste le point de ralliement spatial de l'individu et le fait que les 12 personnes interviewées sont actuellement, du point de vue du logement, géographiquement proches les unes des autres accentue cette structure en étoile. Ceci est nuancé par le fait que, les traits représentant l'ensemble des déplacements au cours de la vie et, par conséquent, à partir de plusieurs logements successifs du fait de déménagements, permettent de voir des centralités secondaires. Sur la deuxième carte, on distingue ainsi un pôle parisien, un pôle dans le Nord-Pas-de-Calais, un pôle en Bretagne intérieure, trois ou quatre dans le quart sud-est de la France, un près de Nice, un autre en Alsace et un de part et d'autre de la frontière germano-polonaise, ce qui est relativement peu étant donné que ces 12 personnes totalisent 101 déménagements ${ }^{21}$.

$\overline{20} \quad$ Dans cette phase de réactivation, bien que l'enquêteur soit présent, l'enquêté s'adresse plus à lui-même qu'à cet enquêteur.

21 Pour un total de 474 années de vie, soit un déménagement tous les 4 ans et 8 mois. 


\section{Carte 1}

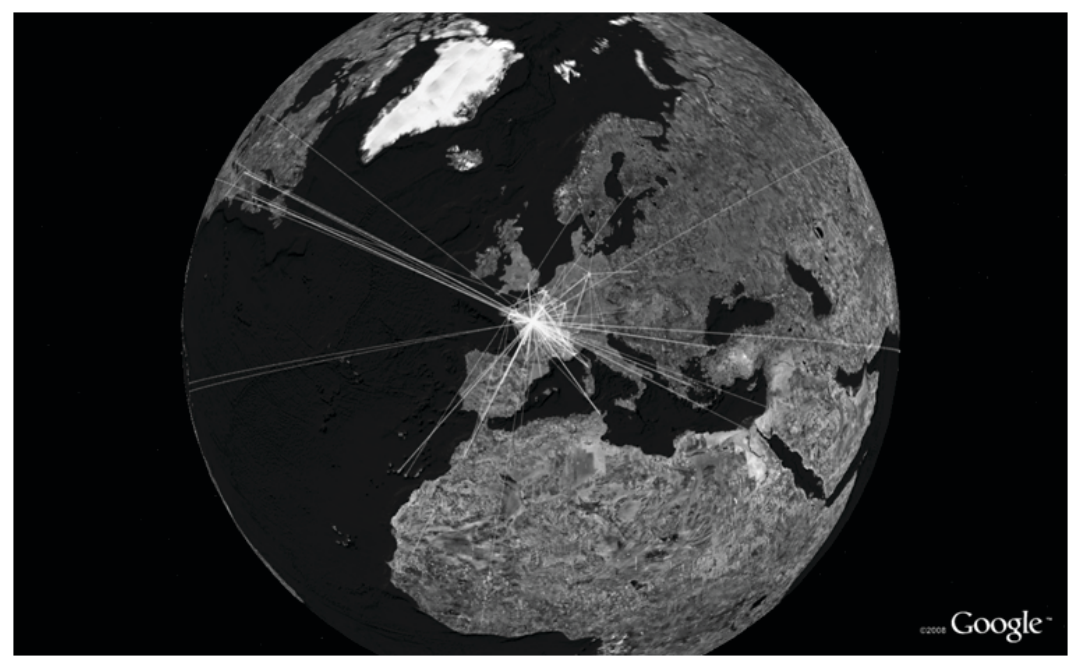

\section{Carte 2}

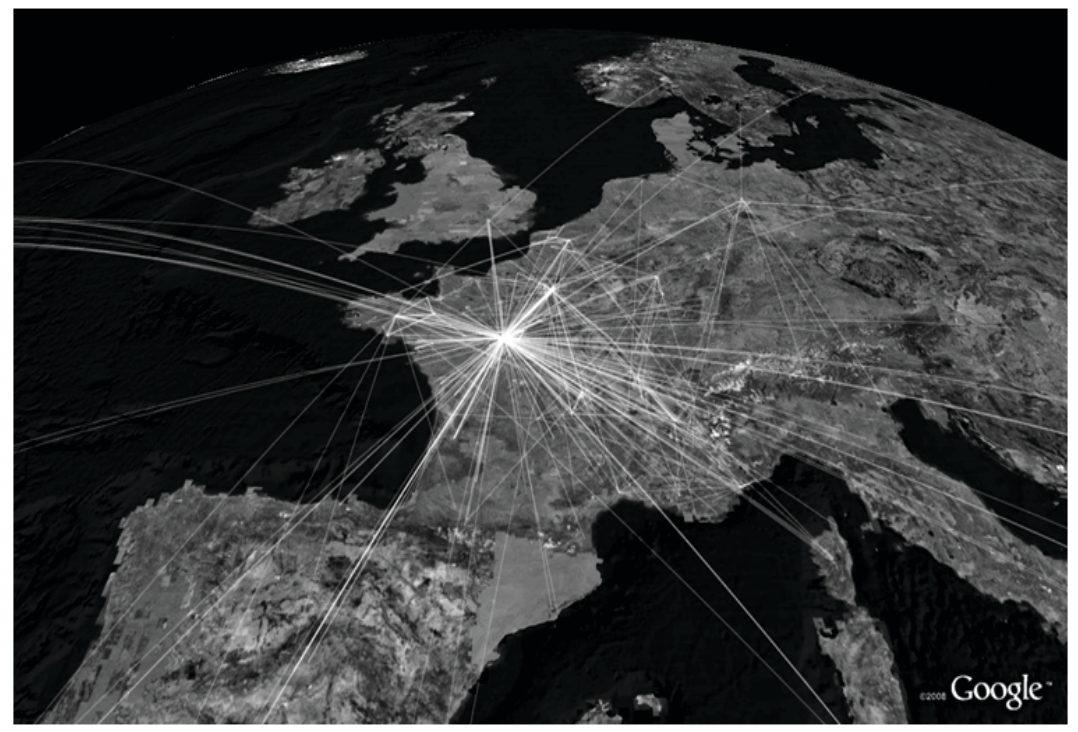

On peut avancer trois hypothèses, non concurrentes, relatives à cette faiblesse.

1. L'hypothèse que, dans le passé, les déplacements étaient 
moins nombreux et, ainsi, les anciens logements ne sont pas au centre d'une étoile d'échelle nationale ou internationale.

2. L'hypothèse que, dans les tranches de vie correspondant au passé, les personnes étant plus jeunes, elles auraient moins voyagé.

3. L'hypothèse que, à l'échelle locale, les déplacements quotidiens sont oubliés ou, en tout cas non restitués lors de l'entretien car jugés de peu d'importance. Il faut ajouter à cela un effet optique de la carte qui ne permet pas de bien distinguer les déplacements locaux sans zoom.

On perçoit des lignes transversales, ne passant pas par Tours, joignant notamment, mais pas uniquement, les pôles secondaires cotés précédemment.

Les échelles plus fines (cartes 3 et 4) permettent de distinguer les trois quartiers d'enquête et d'autres pôles secondaires mais on notera surtout le fait de la densité du maillage, à l'opposé des deux premières cartes. Cependant, ce qui ne peut apparaître sur la carte est le fait que ce maillage est dû, pour beaucoup, à des déplacements de personnes différentes à des moments différents et ne sont pas, sauf exceptions, des représentations de circuits plus complexes que des allers et retours. Ce maillage est donc globalement factice, car il s'agit en fait de plusieurs réseaux hypercentralisés superposés les uns aux autres. D’une façon générale, quelle que soit l'échelle, on ne constate pas de lignes brisées qui montreraient des parcours plus complexes. Le logement (ou le couple logement-lieu de travail) reste bien le lieu central, soit dans la réalité des déplacements, soit dans la reconstruction qui est faite lors du récit de vie spatialisé. Si le logement et son environnement immédiat est effectivement ce qui compte pour l'individu, alors cela justifie, au moins en partie, la possibilité de prise en compte de l'espace du quotidien dans la participation de la population au projet de territoire. Cela est confirmé par l'analyse des spatiogrammes que nous avons construits à partir des récits de vie et qui peuvent se présenter soit à l'échelle de la vie entière, soit à l'échelle temporelle de la tranche de vie. 


\section{Carte 3}

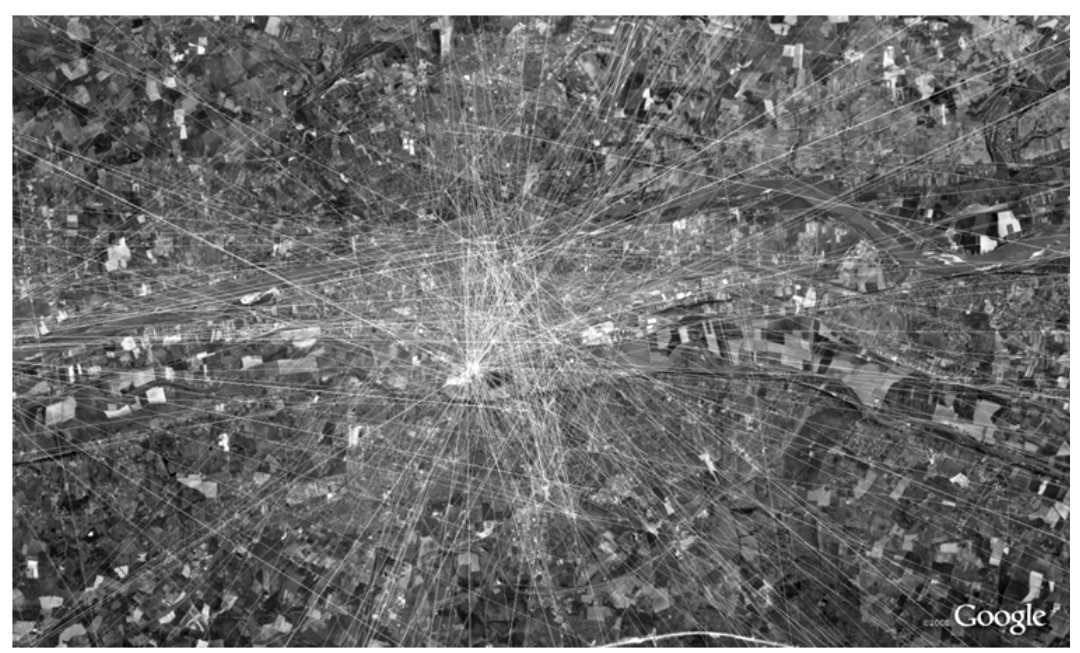

\section{Carte 4}

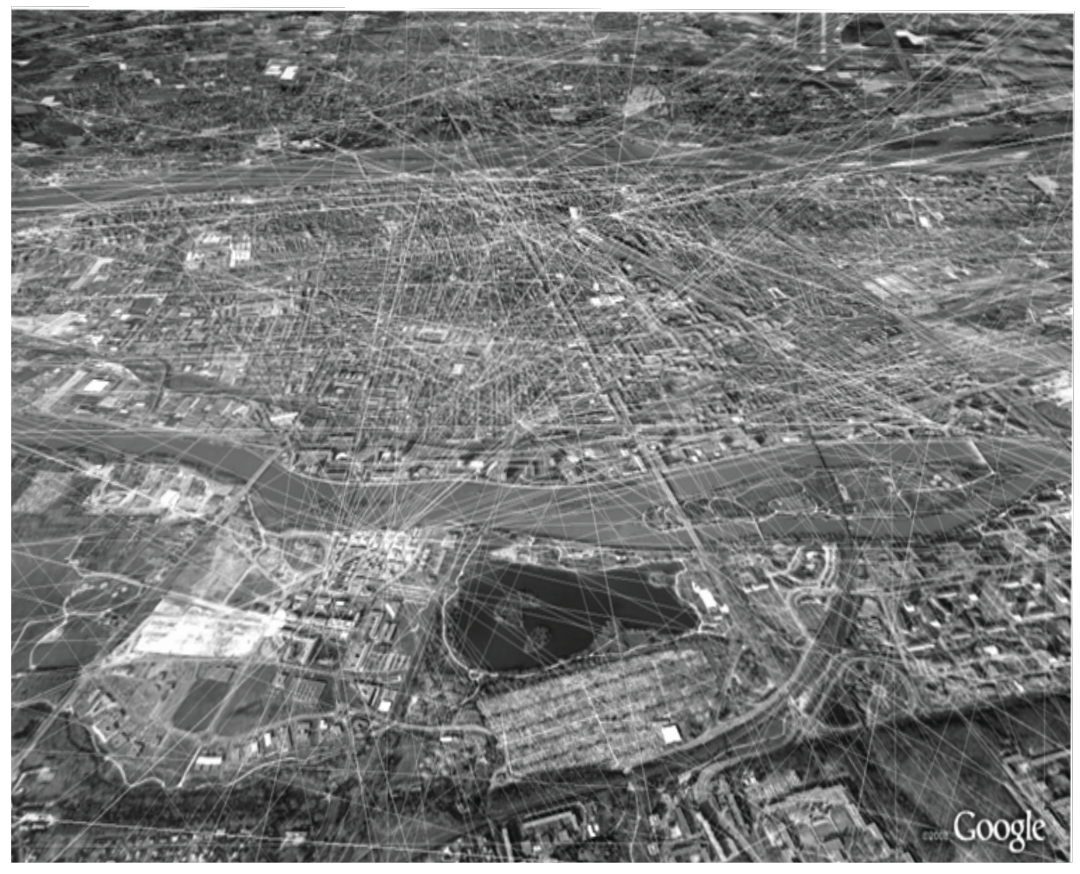


Le traitement du discours des individus, sous forme de tableaux répertoriant les lieux fréquentés, les activités menées en ces lieux, la fréquence de déplacement, etc. permet pour chaque individu d'obtenir une carte de sa spatialité et une série de cartes, chacune représentant une tranche de vie (nous présentons à la carte 5, le spatiogramme ${ }^{22}$ complet de Mlle A et aux cartes 6 et 7 , deux spatiogrammes partiels représentant deux de ses tranches de vie). Ces cartes nuancent le caractère hypercentralisés des réseaux de déplacements, mais montrent néanmoins très peu de trajectoires triangulaires et, a fortiori, plus complexes.

\section{Carte 5}
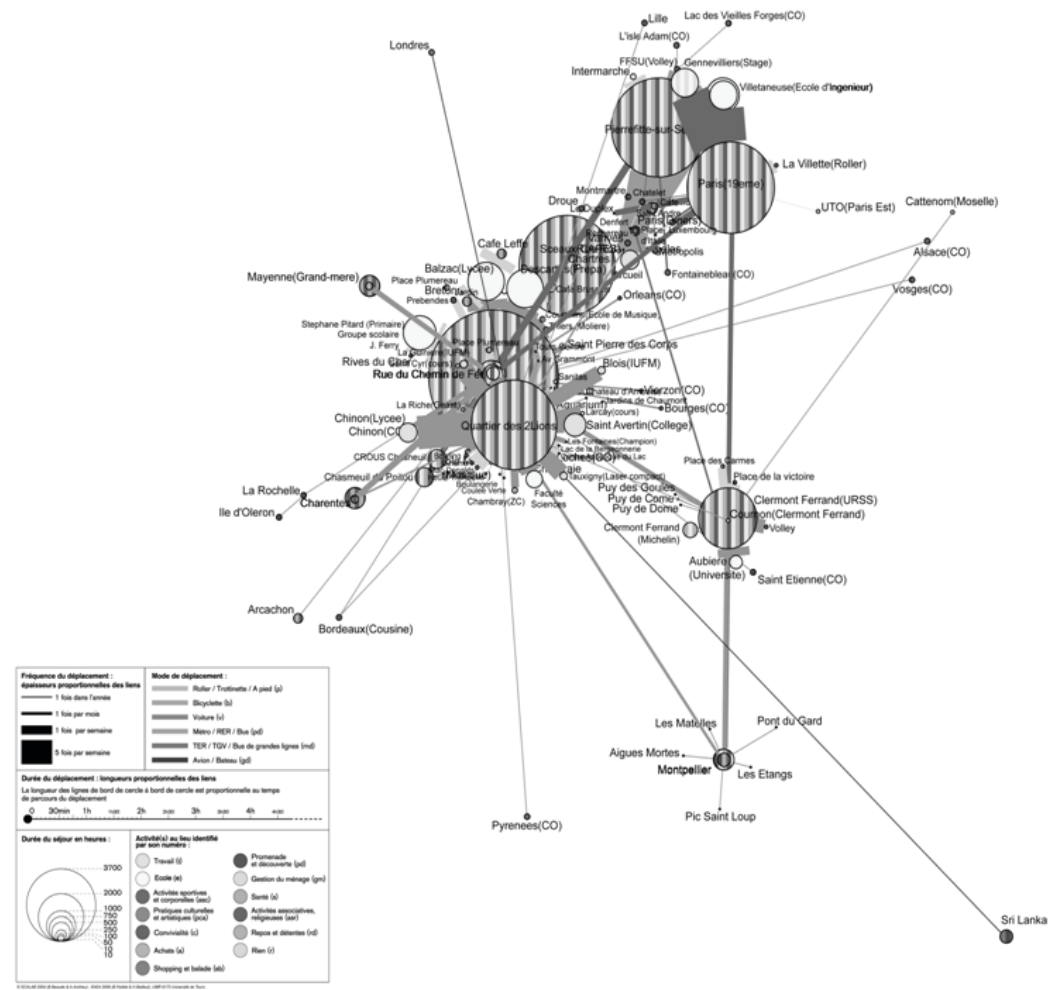

22 Le terme spatiogramme ne réfère pas à ce que Pierre Benghozi ( Le spaciogramme en théorie psychanalytique de couple et de famille ", Dialogue, n ${ }^{\circ}$ 172-2, 2006, p. 5-14) dénomme de la même façon, mais orthographié spaciogramme, qui est un mode de représentation, en psychanalyse, de l'espace domestique et des relations familiales. 
Carte 6

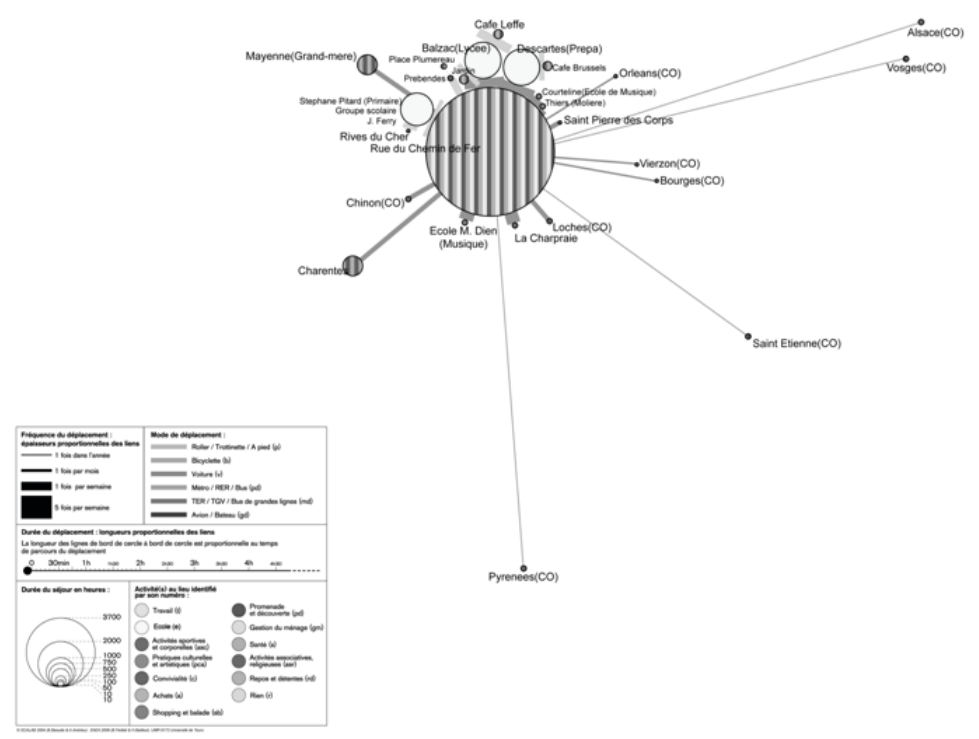




\section{Carte 7}
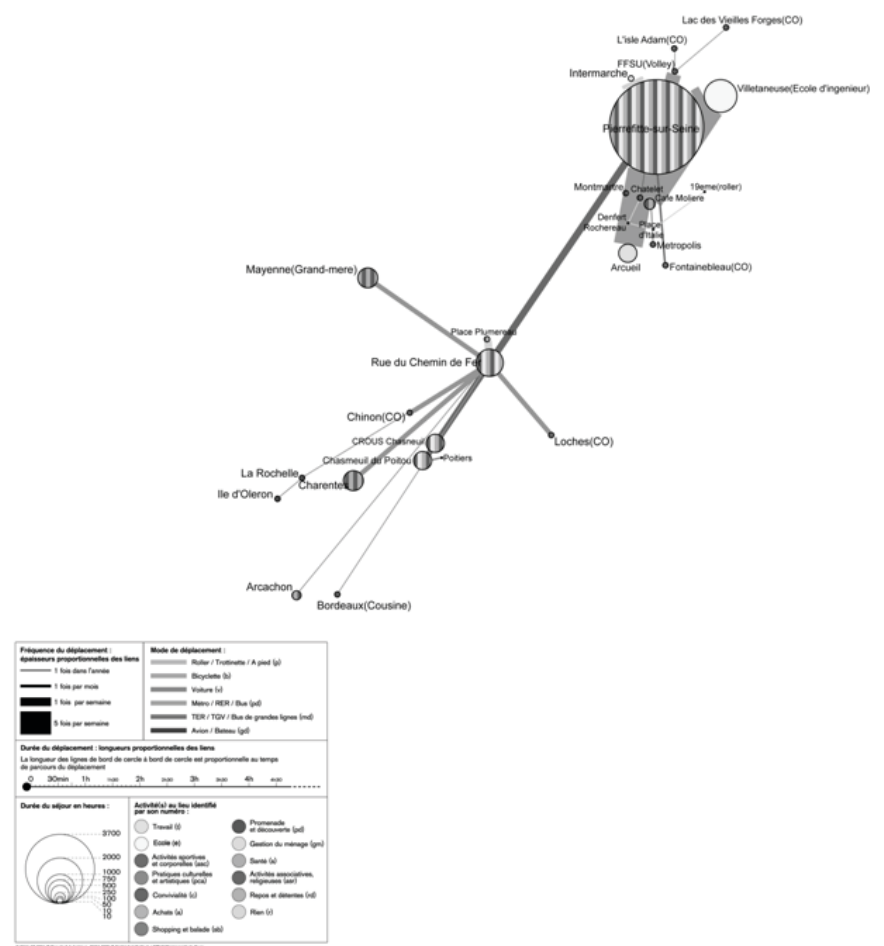

\section{Justifications : de la spatialité à l'habiter}

\subsection{Les différents modes de justification}

Le passage de la spatialité à l'habiter réside, dans les discours donnés à entendre, dans l'ensemble des justifications qui sont, au moins sur le plan formel, amenées pour certains lieux, certains déplacements, voire, plus largement, certains parcours composés de plusieurs lieux et de plusieurs déplacements. Implicitement par le protocole d'enquête, la durée de l'entretien notamment, et explicitement par la demande de l'enquêteur notamment dans la phase de réactivation, l'enquêté est conduit à (se) justifier et ce à deux niveau : celui du contenu du discours en répondant à la question non posée " pourquoi êtes-vous allé là (ou avez-vous fait ceci)? " et celui du discours lui-même : pourquoi (m')avez- 
vous dit être allé là (ou avoir fait ceci)? " : en quoi est-ce important? Ces deux niveaux sont en interaction l'un avec l'autre : l'importance de dire avoir fait quelque chose révèle l'importance de l'action elle-même. Sur un autre plan, le mode d'utilisation d'un espace par quelqu'un révèle tout autant l'espace en question que son utilisateur.

On peut distinguer trois grands cas de justifications qui, dans les discours, s'interpénètrent. Tout d'abord justifier c'est qualifier, mais ce terme est lui-même polysémique et conduit à envisager trois acceptions :

1. qualifier est la reconnaissance d'une caractéristique d'un objet : cet espace est clos, objectivement il l'est;

2. qualifier est la valorisation d'une caractéristique d'un objet : cet espace est clos, objectivement il l'est, mais le qualificateur insiste sur cet aspect et, ce faisant, lui confère une valeur subjectivement posée. Il y a à travers l'adjectif un jugement de valeur (que la suite du discours peut révéler);

3. qualifier est l'attribution d'une valeur à un objet, valeur qu'il n'a pas nécessairement : je trouve cet espace agréable, alors qu'en fait il ne l'est pas objectivement.

Si l'on peut faire cette différenciation formelle, la qualification qu'opère une personne soumise au protocole d'enquête relève des trois modes simultanément : le jugement de fait est en même temps jugement de valeur, puisqu'il est choisi parmi l'ensemble non clos des jugements de fait possibles. Il est donc valorisé par rapport aux autres et c'est ce qui finalement qualifie l'individu, car dans le champ des choix possibles donné par l'espace, le choix relève uniquement de la personne qui ainsi se révèle.

Deuxièmement, justifier c'est expliquer. Dans le sens courant, qualifier un espace est un mode d'explication : je suis en tel lieu parce que j'y trouve de quoi réaliser ce que je souhaite faire. La qualification faite montre l'adéquation ou l'inadéquation entre une volonté (celle de l'individu) et des caractéristiques (celles de l'espace). Nous donnons ici un sens plus restrictif à expliquer. Certains éléments (des paroles entendues, des événements, des ambiances, des sensations...) de la vie passée d'un individu 
expliquent en partie sa situation actuelle. Par exemple, le discours francophile d'un père allemand auprès de sa fille explique en partie la venue de sa fille en France, des années plus tard. De même, des éléments encore virtuels, positionnés dans un futur encore non advenu mais espéré, attendu, craint, fantasmé, souhaité, anticipé, voulu, préparé par un individu permettent d'expliquer en partie sa situation actuelle. Par exemple, tel projet professionnel l'amène à une localisation dans une ville universitaire qui a la probabilité de réaliser ce projet. Cependant, et on le sent tout de suite, ce type d'explication n'est pas satisfaisant : le terme expliquer, ainsi utilisé, relève du langage courant, voire du domaine de la rapidité de langage. Ce type d'explication n'est pas, non plus, suffisant car un élément n'explique pas à lui seul une situation actuelle, ni même une partie de celle-ci et l'on ne peut être sûr du lien qui unit l'élément explicatif de l'élément expliqué. Un discours francophile tenu 40 ans plus tôt ne peut être tenu à lui seul pour la cause d'une localisation actuelle en France. En fait, les éléments passés et ceux qui relèvent d'un futur anticipé préparent tel élément de la situation actuelle. Ainsi, le discours francophile prépare mais n'explique pas au sens précis du terme la venue en France. Par ailleurs, chaque élément pris isolément et chaque composition d'éléments pris conjointement préparent une multitude d'autres situations dont une seule surviendra. Il faut donc se méfier du caractère explicatif de tel élément car celui-ci aurait aussi pu expliquer d'autres situations qui, en d'autres circonstances, auraient pu advenir. En revanche, un faisceau d'éléments (le discours du père, la visite de villes françaises, l'apprentissage de la langue...) aura une valeur explicative plus importante sans pour autant donner une explication pleine et entière. Un faisceau d'éléments préparant est composé d'éléments non connectés (un discours francophile à tel moment et une visite touristique à tel autre moment, ou éventuellement au même moment, et tous les deux préparent, à leur manière, la venue définitive en France) et d'autres qui sont connectés, par exemple, un discours francophile qui prépare une visite touristique en France et l'ensemble prépare la venue définitive en France. 
Ces éléments, préparant la situation actuelle, font en fait augmenter la probabilité que la situation actuelle, celle qui est finalement advenue, advienne. Ainsi, par explication d'une situation ou d'un élément de celle-ci, nous entendons la mise en évidence d'un faisceau d'éléments augmentant la probabilité que la situation ou la partie de situation advienne. Cette préparation se fait par des éléments passés comme par des éléments futurs (par la représentation passée et actuelle de ceux-ci) et de façon directe (ou positive) comme de façon opposée (ou négative) : le discours du père prépare la venue en France parce que ce discours et/ou ce père sont évalués positivement. S'ils avaient été évalués négativement, la préparation se serait faite par opposition (tout sauf la France). Ainsi, par exemple, le ras-le-bol d'un logement trop petit prépare un déménagement vers un logement plus grand.

Troisièmement, justifier c'est rationaliser. Certaines parties des discours sont des rationalisations a posteriori de choix passés ou en cours, voire à venir (à condition qu'ils soient déjà engagés). La rationalisation, qui est une des formes du mensonge à soimême, permet à l'enquêté de justifier des choix desquels il n'est pas ou pas tout à fait satisfait. Ainsi l'individu est cohérent avec lui-même ou plus exactement son discours est cohérent avec l'action décrite dans le discours, mais il n'est pas cohérent avec ses véritables préférences : ainsi, il masque à l'enquêteur (et à lui-même) cette forme d'incohérence. Sur le plan formel et discursif, cette cohérence que l'on peut qualifier de ponctuelle, car elle porte sur un point précis du discours, met à mal la cohérence globale de celui-ci : des contradictions apparaissent. Nous avons développé par ailleurs des exemples de rationalisations a posteriori. Voici, dans ses grandes lignes, l'exemple le plus caractéristique. Les personnes habitant ou ayant longtemps habité en périphérie de la ville ont, de façon assez entière, un discours sur les avantages indéniables d'une telle localisation, omettant radicalement les inconvénients, ce qui peut être synthétisé par la sommation des avantages de la ville qui reste proche (la proximité des services, du lieu de travail, de la diversité, l'accessibilité...) sans en 
subir les inconvénients (le bruit, le stress, l'insécurité, l'embouteillages, la pollution...) et de profiter des avantages de la proche campagne (les espaces ouverts, non pollués, calmes...), sans en subir l'isolement. Ainsi le discours est « je suis en périphérie et j'aime y être " affirmé de telle façon qu'apparaît aberrant tout autre choix de localisation, ce qui est contredit par certains passages de discours de ces personnes qui, tout bien considéré, préfereraient habiter la ville (ou parfois la campagne), mais non cet entre-deux du périurbain. Ce positionnement valorise l'affectif, à travers le choix qui est dit avoir été consciemment et librement pris, et nie les contraintes, notamment financières, qui ont en fait été à l'origine de cette localisation.

Entre qualifications, explications et rationalisations, sont apparues plusieurs modalités de justifications que nous présentons ici succinctement. Tout d'abord, apparaît prioritairement, parce que tout discours, comme reconstruction, est occasion de rationaliser (dans un sens plus large que précédemment) son parcours, des justifications par finalité, reposant sur une raison ou un système de raisons, de type " je suis allé dans telle ville pour y faire les études que j'avais choisi de faire " et des justifications par la causalité, reposant sur une cause ou un système de causes extérieures à l'individu et antérieures à l'action, de type " je suis venu habiter ici parce que j'y avais de la famille ». Ces deux cas sont quasiment interchangeables puisque tout deux révèlent, en fait, l'adéquation ou l'inadéquation évoquée précédemment et, sur un plan formel, on peut aisément transformer un " pour " par un " parce que " et réciproquement, selon que l'on met en avant l'individu et sa volonté (via des raisons) ou l'espace et ses possibilités ou caractéristiques (via des causes) ${ }^{23}$.

Un autre type de justification est celles que l'on peut qualifier de " creuses » ou de purement formelles. La proposition qui la présente contient effectivement au moins un élément de lien entre caractéristique de l'espace et volonté de l'individu, un

23 Sur ce point, nous renvoyons à la lecture de débats en philosophie analytique sur la nature relative des causes et des raisons (par exemple, Donald Davidson, Paradoxes de l'irrationalité, traduit de l'américain par Pascal Engel, Combas, Éditions de l'éclat, 2002). 
" pour" ou un "parce que ", mais les éléments ainsi liés ne le sont que formellement et non logiquement ${ }^{24}$. Peu éloigné de ce modèle de justification, certaines justifications reposent sur des représentations erronées de la réalité. Ainsi, pour justifier de ne pas s'installer à Lyon on a pu entendre : "L'Est, les gens ne sont pas gentils, autour de Lyon ».

Enfin, on notera la fréquence de l'absence de justification qui laisse entrevoir de multiples possibilités : le manque de temps, le peu d'importance de la cause ou de la raison ayant justifié l'action ou ayant amené le fait, à un moment donné d'être là, le peu d'importance d'avoir été là, la volonté de cacher la justification à l'enquêteur...

2.2. Deux cas archétypiques : entre modèle spatial et modèle relationnel

Les deux cas que nous choisissons de présenter ici n'ont pas de valeur représentative de ce que seraient le modèle, la structure ou la forme des espaces vécus de la population en général, ils ont pour utilité de présenter deux archétypes quasi-opposés dans la construction de l'habiter individuel et dans leurs restitutions par les espaces dits. L'un évoque un modèle spatial, l'autre un modèle relationnel. Nous abordons donc le contenu sémantique des justifications apportées, puisque nous en avons précédemment distingué les catégories et les formes.

Le premier exemple est celui de Madame Z, d'origine allemande, 66 ans $^{25}$, qui vit seule en France depuis 1994, année de son départ d'Allemagne consécutif à son divorce. Elle habite actuellement dans le quartier des Deux-Lions à Tours ${ }^{26}$ depuis

24 Ellen Langer, Arthur Blank et Benzion Chanowitz, "The Mindlessness of Ostensibly Thoughtful Action: The role of placebic information in interpersonal interaction ", Journal of Personality and Social Psychology, $\mathrm{n}^{\circ}$ 36, 1978, p. 635-642.

25 Les âges donnés pour les deux exemples sont ceux au moment des entretiens (2006).

26 Le quartier des Deux-Lions est la dernière extension urbaine sur la commune de Tours, extension encore en cours, qui offre un espace mixte (activités tertiaires et secondaires, logements de différents types, université, écoles...) dans un cadre alliant modernité architecturale et traitement paysager. 
octobre 2003, et s'y sent visiblement bien. Sa localisation aux Deux-Lions est en partie déterminée par ce que propose ce quartier en termes d'aménités et d'urbanité et en partie par son parcours personnel, son cheminement depuis plus de six décennies.

Le premier point qui explique la présence ici de $\mathrm{Z}$ est la francophilie de son père qui, bien que nazi ayant œuvré en France pendant la guerre, lui a enseigné, peut-être inculqué, que la France est le pays d'une certaine culture privilégiée, en tout cas d'une culture à connaître, ce qui, pour $\mathrm{Z}$ passera par l'apprentissage de la langue française à partir de l'adolescence. Plus tard, elle sera jeune fille au pair, à trois reprises, à Paris, dans une famille de la bourgeoisie parisienne soucieuse de mettre en œuvre la réconciliation franco-allemande. Entre-temps, sa connaissance de la langue française lui permet de participer à des échanges liés au jumelage entre sa ville Wolfsburg et Marignane. Elle découvre donc une façon de vivre qui lui plaît :

la raison pour laquelle je suis partie définitivement en France il y a 11 ans, c'est que j'avais déjà ressenti ça quand j'étais jeune, cette façon différente de vivre, une certaine légèreté dans l'esprit des Français.

Le deuxième point qui explique sa présence à Tours est son départ d'Allemagne, à 54 ans, suite à son divorce sans que celui-ci explique précisément sa venue en France. On a là une pseudo-justification : son divorce peut expliquer son départ de chez elle, non d'Allemagne, ni a fortiori le fait d'aller quelque part en particulier. On a ensuite une justification " creuse ", insuffisante : ses filles étant autonomes depuis longtemps, elle peut choisir. Elle prend une carte de France et procède par élimination, en ce qui concerne les grandes régions :

le Nord, la Normandie, c'est joli, mais pas en hiver... La Bretagne non plus. Le sud-ouest, c'est trop loin de ma famille. Le sud, c'est trop chaud. L'est, les gens ne sont pas gentils, autour de Lyon. Alsace, trop proche de l'Allemagne, Lorraine non plus, Belgique non plus. Paris, jamais.

Bref, il ne reste plus, sans doute après d'autres éliminations implicites, que le centre du pays. Elle va donc choisir une ville, en fonction de deux critères, assez complémentaires : la taille de 
la ville et la présence d'une université. Ce n'est que quand elle est arrivée à Tours qu'elle se souvient d'y être déjà venue, une journée pour visiter les châteaux de la Loire et d'avoir lu un livre en allemand sur le " charme discret de la bourgeoisie tourangelle». Faut-il y voir une explication du choix final entre les deux villes qui restaient en balance : Tours et Orléans?

Troisièmement, sa présence dans le quartier des Deux-Lions, en bordure du Cher, est le croisement de deux séries de justifications. La première est en rapport avec son précédent logement, trop petit, avec trop peu d'ouverture visuelle sur l'extérieur. Aussi le logement aux Deux-lions est comparativement mieux. La seconde série est en lien avec son enfance. Comme pour le précédent logement, un point positif est le caractère neuf du logement, c'est-à-dire son côté pratique et surtout moderne. Mais cette modernité s'exprime d'abord à l'échelle du quartier des Deux-Lions. La justification qu'elle apporte alors est ancrée dans ses souvenirs, notamment ceux concernant la ville de Wolfsburg. Cette idée de modernité est très largement développée lorsqu'elle décrit cette ville : " j'ai habité 26 ans dans une ville moderne qui avait été créée par Hitler en 38 pour la construction de Volkswagen [...], très moderne, très riche ». Pourtant, plus que la modernité, ce sont le cadre de vie et sa qualité, en accord avec le respect de l'environnement, du paysage.

Ils n'ont pas touché à la forêt, pour agrandir la ville, pour faire les différents quartiers, ils ont laissé tous les arbres. C'étaient les responsables de l'usine Volkswagen qui ont demandé à l'administration : si jamais vous créez plus de quartier, parce que l'on recrute de plus en plus de travailleurs d'Allemagne et d'ailleurs, il ne faut pas toucher à la forêt, il faut créer un habitat qui la respecte. C'était dans les années 50 , et déjà, c'était exemplaire. Sous les arbres ils ont donc construit de petites maisons, des immeubles... tellement adaptés aux paysages, avec des aires de jeux déjà et tout le confort [...] Comme l'usine de Volkswagen avait beaucoup de recettes, ils ont investi dans les beaux-arts. On a eu les meilleures expositions de toute l'Allemagne sur place, parce que c'était sponsorisé. Juste avant mon départ, ils ont construit un grand musée, plus grand que le palais du Vinci ${ }^{27}$, tout en verre. Et pour l'inauguration, il y avait les œuvres de Fernand Léger, et d'autres artistes modernes

$\overline{27} \quad$ Le Vinci est le Palais des Congrès de Tours, conçu par Jean Nouvel. 
étrangers. Ça a coûté une fortune. On a eu un planétarium qui projetait la voûte céleste. Volkswagen a offert l'appareil à la ville de Wolfsburg qui a été obligée de construire la coupole et le bâtiment [...]. On avait un théâtre ultramoderne, laid de l'extérieur mais fonctionnel à l'intérieur, pour les concerts, il y avait la meilleure acoustique. Pour le théâtre la scène pouvait se tourner... Tout ça, planté sur une colline vers la forêt. Au milieu de la forêt, il y avait un hôpital, un grand complexe, et à droite du théâtre ils ont mis sur un terrain vert, sur le gazon, le planétarium en centre-ville. Les gens sont venus de toutes les directions pour admirer ça. Et plus tard, il y a eu un centre aquatique, beaucoup plus luxueux.

Cette ville ne compte pourtant que 38000 habitants en 1954 et 120000 aujourd'hui

Le second cas est celui de C, une jeune femme de 26 ans, non mariée mais vivant en couple, sans enfant. Son histoire, plus courte et moins dense que celle de $Z$ est peut-être plus ordinaire. Sa spatialité se construit autour de deux pôles, à partir de l'âge de 4 ans, c'est-à-dire lorsque ces parents divorcent et habitent dans deux communes relativement proches l'une de l'autre, dans le Nord-Pas-de-Calais. Ainsi, très classiquement, les lieux symbolisés par la mère sont ceux de l'école puis du collège, du quotidien dans ce qu'il peut avoir de terne, par opposition au père qui représente les lieux de vacances et de loisir, où les contraintes sont nettement moindres. Cette différence d'évaluation affective est alourdi par le fait que les moments auprès du père sont moindre, en durée, que ceux passés chez ou auprès de la mère. Sur le plan relationnel, à l'école comme dans la famille élargie, $\mathrm{C}$ se sent à part, on ne peut savoir si elle l'est réellement, car elle est, chaque fois " la seule fille de parents divorcés ». Son premier logement se situe à mi-distance de ceux de ses parents comme si elle ne voulait pas avoir à choisir ou à montrer une préférence. Les circonstances font que, à peu près simultanément, alors qu'elle a 21 ans, ses parents déménagent, l'un à Bordeaux, l'autre près de Nice. Plus tard, elle déménagera elle-même pour venir s'installer avec son compagnon à Tours. On note que, pour C, l'espace est partagé, d'abord temporellement quand elle dépend directement de ses parents auprès de qui elle se partage, 
puis géographiquement par son positionnement presque exactement sur la bissectrice qui sépare les deux points représentant les lieux de vie principaux de ses parents. Un nouveau déménagement est envisagé, mais pour cause de mutation de son compagnon, et cette bissectrice sera alors peut-être brisée.

\subsection{Modèle spatial vs modèle relationnel}

Il apparaît clairement que lors de ces deux entretiens cohabitent et interagissent des éléments qui sont de l'ordre de l'a-spatial et des éléments spécifiquement, idéellement ou concrètement spatiaux. Ce qui ressort principalement est le fait que chez Z, les justifications sont principalement mais non uniquement à caractère spatial. D'une part, elle traduit avant tout sa rupture par une mise à distance qui se concrétise géographiquement par plusieurs centaines de kilomètres, mais surtout son installation actuelle est la tentative pour retrouver un modèle de ville dont elle a objectivement souvenir et pour lequel elle a une évaluation, y compris sur le plan affectif, très positive. Quelle que soit la ou les raisons de ce choix en partie rationnel en partie affectif pour ce type de ville et les raisons de la recherche d'exemple relatif à ce modèle, il apparaît que l'espace dit de la personne peut, en partie au moins, contribuer à préfigurer le projet de territoire. Chez Z, d'ailleurs, cela est aussi lié à un mode de fonctionnement de la ville, fondé, en ce qui concerne les déplacements, en vélo et à pied dans des espaces végétalisés, non artificiels.

En revanche, dans le cas de $\mathrm{C}$, on a très peu de détails de l'espace physique et visuel, sans que pour autant celui-ci soit inexistant dans son espace vécu (il est pourvoyeur de travail, par exemple) mais ici la mise à distance ou plus exactement la bonne distance vis-à-vis de ses parents n'est pas relayée par une dimension relative aux contenus des espaces et des lieux quelle a connu, que ce soit comme modèle ou comme contre-modèle.

$Z$ organise bien la relation entre la série de tranche de vie et ce que chacune apporte et la situation actuelle : elle se sert de ce que donne à utiliser l'espace actuellement fréquenté pour répondre à ses aspiration héritées de son passé, de son histoire. $A$ 
contrario, C laisse penser que sa détermination spatiale est avant tout relationnelle et qu'en second lieu interviennent des caractéristique d'ordre spatial. Par ailleurs, chez $\mathrm{C}$ les caractéristiques d'ordre spatial semblent utilisées hic et nunc, c'est-à-dire ce que propose concrètement un lieu considéré en fonction des besoins du moment, tandis que, chez Z, la recherche n'a pas été celle d'aménités additionnées les unes aux autres mais celle d'un modèle de ville, même si l'aboutissement actuel suppose des concessions par rapport au modèle de la ville moderne et respectueuse de la nature. Par exemple, le quartier des Deux-Lions donne une image, un paysage qui masque que la construction de ce quartier n'a pas été respectueuse des principes écologiques mais l'a rapproché de Wolfsburg :

[...] Quand je suis arrivée en 1994, mes première promenades, disons, étaient à partir de 1995. Et là, avant les constructions pour canaliser le Cher, j'ai vu sur le bord du Cher, avant le pont de Saint-Sauveur, encore une vraie niche écologique. Il y avait deux îles dans le Cher et une rive toute plate, toujours inondée, où il y avait des pêcheurs et des oiseaux rares. Le Cher faisait un tournant. J'ai été scandalisée quand les pelleteuses sont arrivées et qu'ils ont mis du remblai. J'ai écrit à ma famille en Allemagne, qu'ils étaient en train d'endiguer le Cher, de faire disparaître la dernière niche écologique de Tours. J'étais scandalisée.

\section{Conclusion}

La recherche menée nous donne donc quelques indications sur le rapport qu'entretiennent les individus envers l'espace, par le moyen du récit de vie spatialisé (les pratiques des différents lieux qui composent cet espace, mais aussi les souvenirs, les attentes...). Au-delà des connaissances que peuvent avoir des habitants à propos de l'urbain, au-delà de la capacité à abstraitiser, modéliser, généraliser les connaissances partielles que l'on a de villes particulières, au-delà de la capacité à s'exprimer là-dessus, il apparaît que l'on peut distinguer deux idéaux-types en matière d'habiter, c'est-à-dire de spatialité argumentée.

Le premier est celui des personnes qui tirent et forgent leur conception de l'espace à partir de celui-ci, de ses caractéristiques 
et des lieux qui le composent (et l'évaluation qui va de pair ainsi que les pratiques qui en découlent), de l'expérience qu'ils en ont eue dans le passé, tandis que le second réfere aux personnes qui se contentent d'utiliser les caractéristiques de l'espace là où ils sont en fonction de leurs besoins. Parmi ces personnes, certaines l'utilisent en fonction de besoins d'ordre socioéconomique, ce qui correspond finalement à une représentation de ville assez floue et abstraite qui serait celle correspondant à la ville efficace. D'autres vont utiliser l'espace en fonction de besoins qui sont, comme chez C, d'ordre psychoaffectif et leur espace est alors structuré par le positionnement géographique de personnes ou d'éléments concrets évaluées affectivement et non par des caractéristiques spatiales, celles sur lesquelles les aménageurs, urbanistes, paysagistes, développeurs peuvent plus aisément intervenir. Le cas de C est emblématique : elle se localise sur une droite qui lui permet d'être à bonne distance de chacun de ses parents, sans tenir véritablement compte des aménités procurées par cette ligne (même si, bien évidemment, elle répond nécessairement à ses besoins d'ordre socioéconomique). On pourrait la qualifier de plus géométrique que géographique. Son cas est d'autant plus intéressant qu'elle a une approche relativement négative de ses relations sociofamiliales.

Cela signifie que l'espace vécu, englobant l'espace dit, ne suffit pas en soi pour être pris en compte, via la participation, dans la construction d'un projet de territoire, dont les limites mêmes ne correspondent pas aux spatialités et moins encore à l'habiter, même si ce territoire répond aux besoins les plus concrets; il faut aussi avoir connaissance des modalités de construction de cet habiter. Il ne s'agit pas de remettre en cause la participation du public dans l'élaboration d'un projet de territoire ou d'un projet d'urbanisme, mais de ne pas lui donner une valeur opératoire plus forte qu'elle n'a. 


\section{Bibliographie}

Bailleul, Hélène, Benoît Feildel, Lise Gaignard et Denis Martouzet, «La carte : fonctionnalité transitionnelle et dépassement du récit de vie ", Nature, Sciences, Sociétés, 2009 (à paraître).

Benghozi, Pierre, «Le spaciogramme en théorie psychanalytique de couple et de famille ", Dialogue, n 172-2, 2006, p. 5-14.

Berque, Augustin, "Qu'est-ce que l'espace de l'habiter? ", Colloque, IUP, 11-12 mai 2006.

Collectif EHEA, Espaces habités, espaces anticipés, rapport de recherche UMR CITERES pour le compte de l'Agence nationale de la recherche, 2008.

Davidson, Donald, Paradoxes de l'irrationalité, traduit de l'américain par Pascal Engel, Combas, Éditions de l'éclat, 2002.

Feldman, Robert, James Forrest et Benjamin Happ, «Self-presentation and verbal deception: Do self-presenter lie more? ", Basic and applied social psychology, n 24, 2002, p. 163-170.

Gwiazdzinski, Luc (dir.), La ville 24 heures sur 24 : regards croisés sur la société en continu, La Tour d'Aigues, Éditions de l'Aube, Paris, DATAR, 2003.

Heidegger, Martin, Essais et conférences, Paris, Gallimard, 1958.

Kaufmann, Jean-Claude, Linvention de soi, une théorie de l'identité, Paris, Armand Colin, 2004.

Langer, Ellen, Arthur Blank et Benzion Chanowitz, «The Mindlessness of Ostensibly Thoughtful Action: The role of placebic information in interpersonal interaction ", Journal of Personality and Social Psychology, $\mathrm{n}^{\circ} 36,1978$, p. 635-642.

Lévy, Jacques et Michel Lussault (dir.), Dictionnaire de la géographie et de l'espace des sociétés, Paris, Belin, $1033 \mathrm{p}$.

Loftus, Elizabeth, "Our changeable memories : Legal and practical implications ", Nature Review Neurosciences, n 4, 2003, p. 231-234.

Martouzet, Denis, « La perception de l'urbain par la population martiniquaise et conséquences urbaines et spatiales ", Annales de géographie, n 623, janvier-février 2002, p. 73-85.

Martouzet, Denis, « Methodological Individualism and affective relationship to the city ", communication au $2^{d}$ World Schools Congress, Planning for diversity and multiplicity, Mexico City, 12-16 juillet 2006.

Martouzet, Denis, «Le rapport affectif à la ville : premiers résultats ", dans Thierry Paquot, Michel Lussault et Chris Younès (dir.), Habiter, le propre de l'homme. Villes, territoires et philosophie, Paris, La Découverte, 
2007, p. 171-192.

Martouzet, Denis, «Le rapport affectif à la ville : positionnement théorique et épistémologique ", Praxis Revue électronique en aménagement, [en ligne] http://www.revue-praxis.fr/document.php?id=117, site consulté le 5 juillet 2008 .

Martouzet, Denis, «Le rapport affectif à la ville : discours et rationalisations », Séminaire Individu et ville, UMR CITERES, Tours, 19-20 mars 2007.

Paquot, Thierry, Michel Lussault et Chris Younès (dir.), Habiter, le propre de l'homme. Villes, territoires et philosophie, Paris, La Découverte, 2007.

Tabary, Jean-Claude, " Mémoire et autonomie ", dans Élie Bernard-Weil et Jean-Claude Tabary (dir.), Praxis et cognition, Lyon, L'Interdisciplinaire, 1992, p. 95-102. 